\title{
Optical Spectra of the Type la Supernova 1998aq
}

\section{Citation}

Branch, David, Peter Garnavich, Thomas Matheson, E. Baron, R. C. Thomas, Kazuhito Hatano, Peter Challis, Saurabh Jha, and Robert P. Kirshner. 2003. "Optical Spectra of the Type la Supernova 1998aq." The Astronomical Journal 126 (3): 1489-98. https://doi.org/10.1086/377016.

\section{Permanent link}

http://nrs.harvard.edu/urn-3:HUL.InstRepos:41417341

\section{Terms of Use}

This article was downloaded from Harvard University's DASH repository, and is made available under the terms and conditions applicable to Other Posted Material, as set forth at http:// nrs.harvard.edu/urn-3:HUL.InstRepos:dash.current.terms-of-use\#LAA

\section{Share Your Story}

The Harvard community has made this article openly available.

Please share how this access benefits you. Submit a story.

\section{Accessibility}


OPTICAL SPECTRA OF THE TYPE Ia SUPERNOVA 1998aq

\author{
David Branch, ${ }^{1}$ Peter Garnavich, ${ }^{2}$ Thomas Matheson, ${ }^{3}$ E. Baron, ${ }^{1}$ R. C. Thomas, ${ }^{1}$ Kazuhito Hatano, ${ }^{4}$ \\ Peter Challis, ${ }^{3}$ Saurabh Jha, ${ }^{3}$ and Robert P. Kirshner ${ }^{3}$ \\ Received 2003 April 14; accepted 2003 May 15
}

\begin{abstract}
We present 29 optical spectra of the normal Type Ia SN 1998aq, ranging from 9 days before to 241 days after the time of maximum brightness. This spectrocopic data set, together with photometric data presented elsewhere, makes SN 1998aq one of the best observed Type Ia supernovae at optical wavelengths. We use the parameterized supernova synthetic-spectrum code SYNOW to study line identifications in the early photospheric-phase spectra. The results include evidence for lines of singly ionized carbon, at ejection velocities as low as 11,000 $\mathrm{km} \mathrm{s}^{-1}$. Implications for SN Ia explosion models are discussed.
\end{abstract}

Key words: supernovae: general — supernovae: individual (SN 1998aq)

\section{INTRODUCTION}

The discovery in a relatively nearby galaxy of a Type Ia supernova (SN Ia), more than a week before its time of maximum brightness, presents an observational opportunity that should not be missed. A few days after the discovery of SN 1998aq in NGC 3982 on 1998 April 13 by the UK Supernova/Nova Patrol (Hurst, Armstrong, \& Arbour 1998), photometric and spectroscopic observations undertaken at the F. L. Whipple Observatory (FLWO) revealed that the supernova was a Type Ia whose brightness was on the rise (Garnavich et al. 1998). Subsequent observations resulted in SN 1998aq becoming one of the best observed SNe Ia at optical wavelengths. The Hubble Space Telescope has been used by Saha et al (2001; see also Stetson \& Gibson 2001) to make a Cepheid-based determination of the distance to NGC 3982, so that SN 1998aq can be added to the list of $\mathrm{SNe}$ Ia that are used to calibrate the extragalactic distance scale.

A preliminary report of the photometric observations of SN 1998aq has been given by Boffi \& Riess (2003), and the final results will appear elsewhere (Riess et al. 2003). SN $1998 \mathrm{aq}$ reached a maximum brightness of $B=12.39$ on 1998 April 27. The value of $\Delta m_{15}$, the decline of the blueband magnitude during the first 15 days after maximum (Phillips 1993), was 1.14, a typical value for a normal SN Ia. SN 1998aq was photometrically normal, except that some of its broadband colors were unusually blue; e.g., $B-V=-0.18$ at the time of $B$ maximum.

\section{OBSERVATIONS}

Low-dispersion spectra of SN 1998aq were obtained with the FAST spectrograph (Fabricant et al. 1998) on the Tillinghast $1.5 \mathrm{~m}$ telescope at FLWO. The FAST uses a $2688 \times 512$ Loral CCD with a spatial scale of 1 ."1 pixel $^{-1}$ in the binning mode used for these observations. Details of the

\footnotetext{
${ }^{1}$ Department of Physics and Astronomy, University of Oklahoma, 440 West Brooks Street, Norman, OK 73019.

2 Department of Physics and Astronomy, 225 Nieuwland Science Hall, University of Notre Dame, Notre Dame, IN 46556.

${ }^{3}$ Harvard-Smithsonian Center for Astrophysics, 60 Garden Street, Cambridge, MA 02138.

${ }^{4}$ Department of Astronomy, School of Science, University of Tokyo, Tokyo, Japan.
}

exposures are given in Table 1. The data were reduced in the standard manner with IRAF $^{5}$ and our own routines. The spectra were optimally extracted (Horne 1986). Wavelength calibration was accomplished with $\mathrm{HeNeAr}$ lamps taken immediately after each SN exposure. Small-scale adjustments derived from night-sky lines in the SN frames were also applied. Spectrophotometric standards are listed in Table 1. We attempted to remove telluric lines using the well-exposed continua of the spectrophotometric standards (Wade \& Horne 1988; Matheson et al. 2001). The spectra were, in general, not taken at the parallactic angle (Filippenko 1982). For the spectra modeled in this paper, though, the air mass was low $(<1.2)$. We estimate that the relative spectrophotometry is accurate to better than $5 \%$.

The spectra, corrected for the $z=0.003699$ redshift of NGC 3982 and labeled by the epoch in days with respect to the date of maximum brightness in the $B$ band (1998 April 27 ), are displayed in Figure 1. The spectra are those of a normal SN Ia. The early spectra, from -9 to 7 days (Fig. 1a), contain the characteristic deep $\mathrm{Si}$ II absorption near $6100 \AA$ and the distinctive S II W-shaped feature from about 5200 to $5500 \AA$ A. The spectra between 19 and 91 days (Figs. $1 a$ and $1 b$ ) are those of a SN Ia making the transformation from the photospheric to the nebular phase. By 211 days (Fig. 1c) the transformation is complete.

In Figure 2, four spectra of SN 1998aq are compared with those of another well observed event, SN 1994D (Patat et al. 1996). The similarity of the spectra at -8 days (Fig. 2a) is impressive, considering that premaximum spectra of SNe Ia exhibit considerable diversity (e.g., Hatano et al. 2000; Li et al. 2001). At -8 days the main difference is that SN 1998aq has a peak near $4600 \AA$ that is not present in SN 1994D; otherwise, practically all of the features, including the weak ones, are quite similar. At 4, 19, and 54-55 days the spectra are also similar, although they are distinguishable, e.g., near $4100 \AA$ at 4 days and near $5400 \AA$ at 19 and 54-55 days.

In Figure 3 the blueshifts, expressed in kilometers per second, of the absorption near $6100 \AA$ produced by Si II $\lambda 6355$ in the early spectra of SNe 1998aq and 1994D are

\footnotetext{
${ }^{5}$ IRAF is distributed by the National Optical Astronomy Observatory, which is operated by the Association of Universities for Research in Astronomy (AURA), Inc., under cooperative agreement with the National Science Foundation.
} 


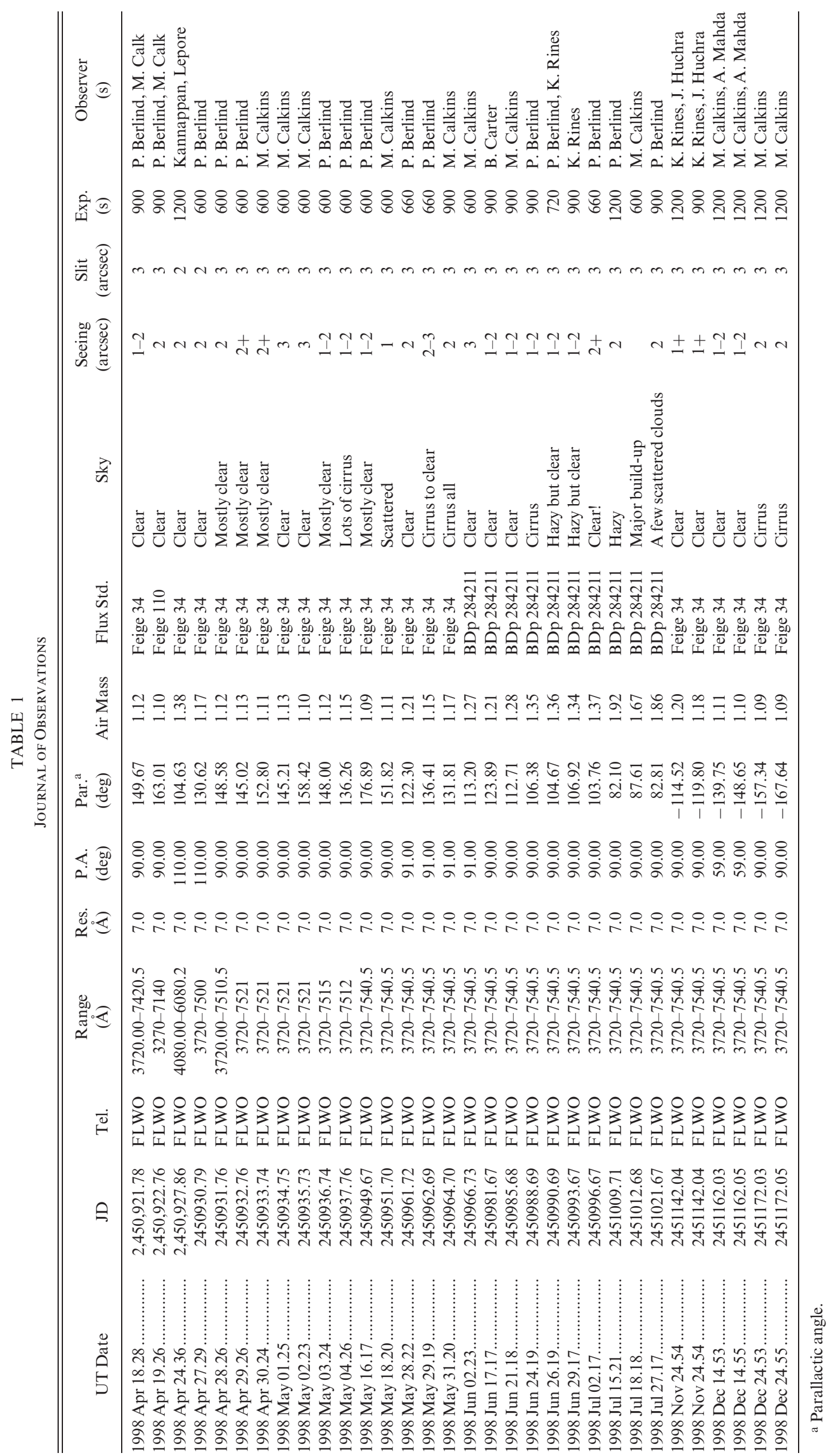




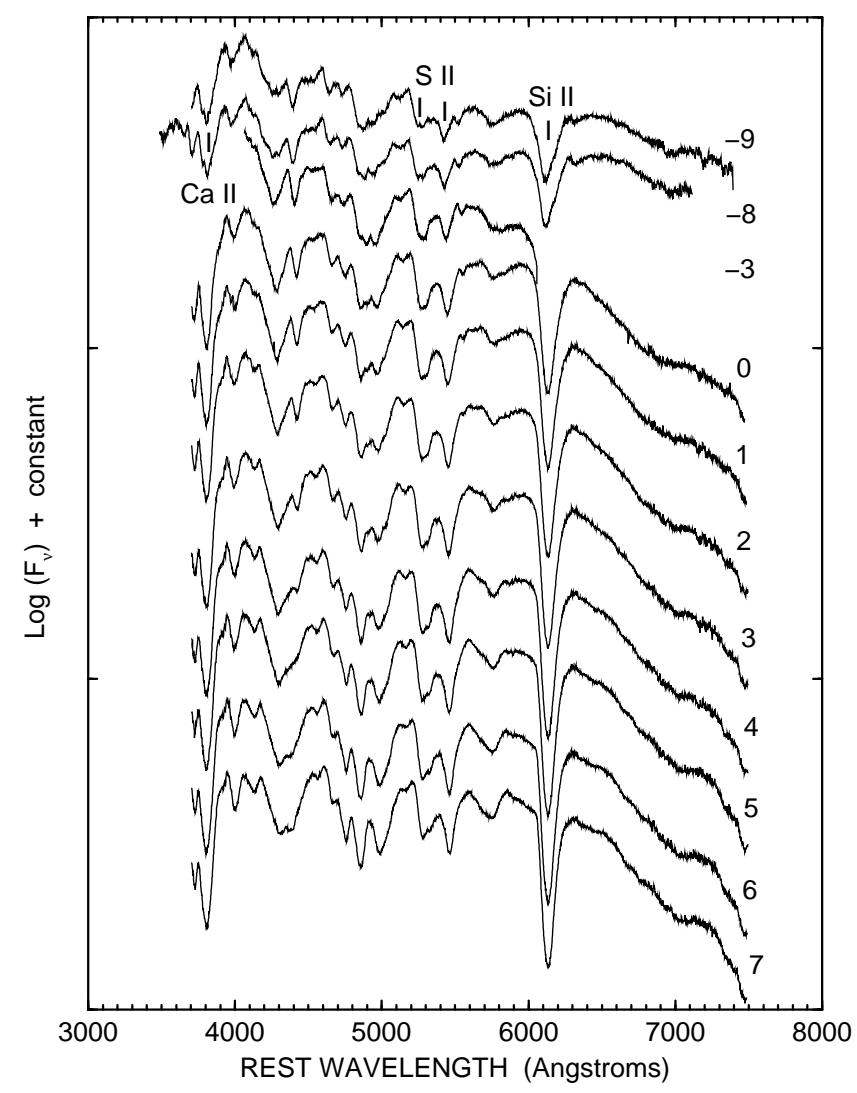

FIG. $1 a$

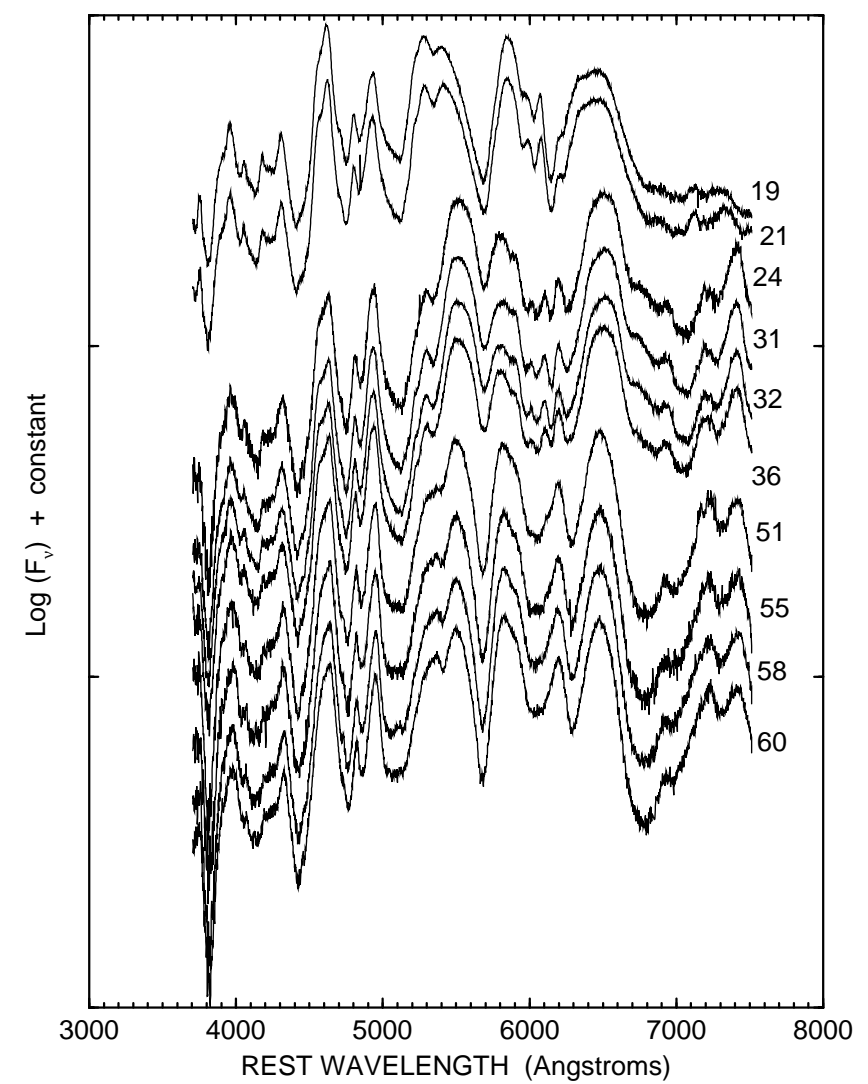

FIG. $1 b$

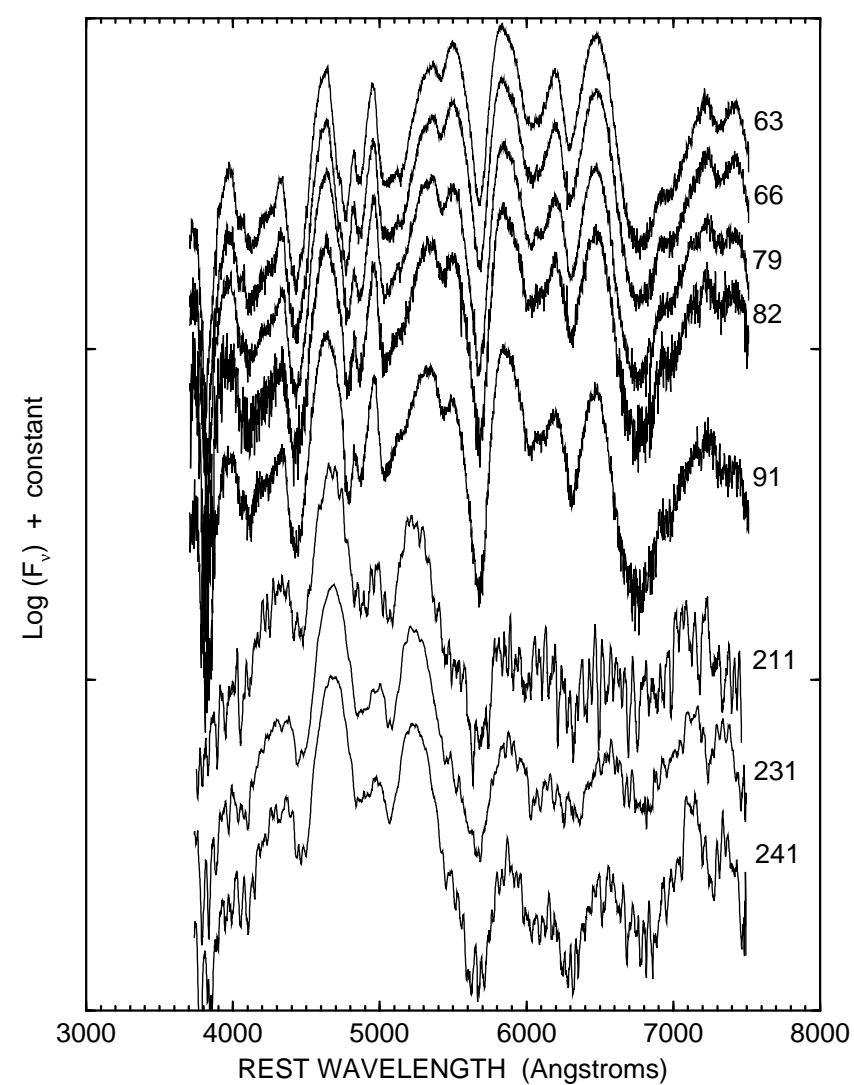

FIG. $1 c$

FIG. 1.-Spectra of SN 1998aq. Epochs are in days with respect to the date of maximum brightness in the $B$ band, 1998 April 27 . The vertical displacement is arbitrary. All spectra shown in this paper have been corrected for the redshift of NGC 3982, $z=0.003699$. No correction for interstellar reddening has been applied. 


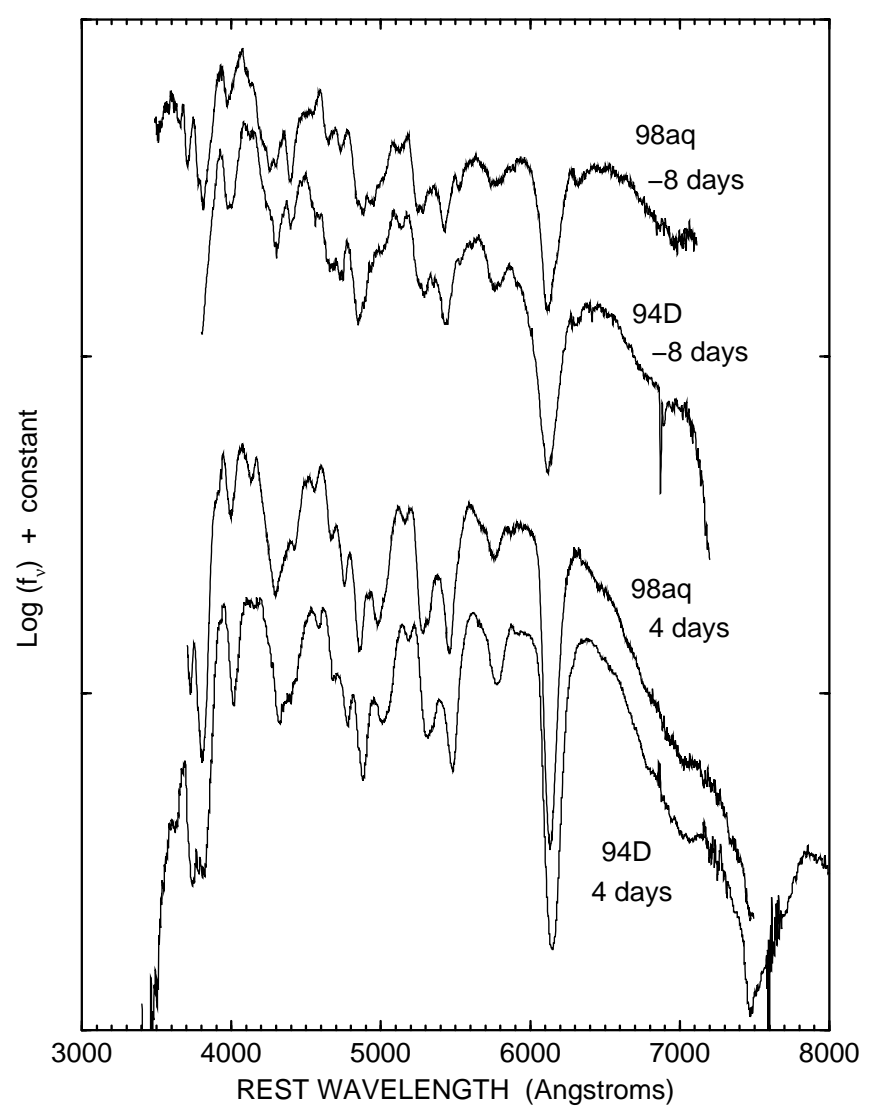

FIG. $2 a$

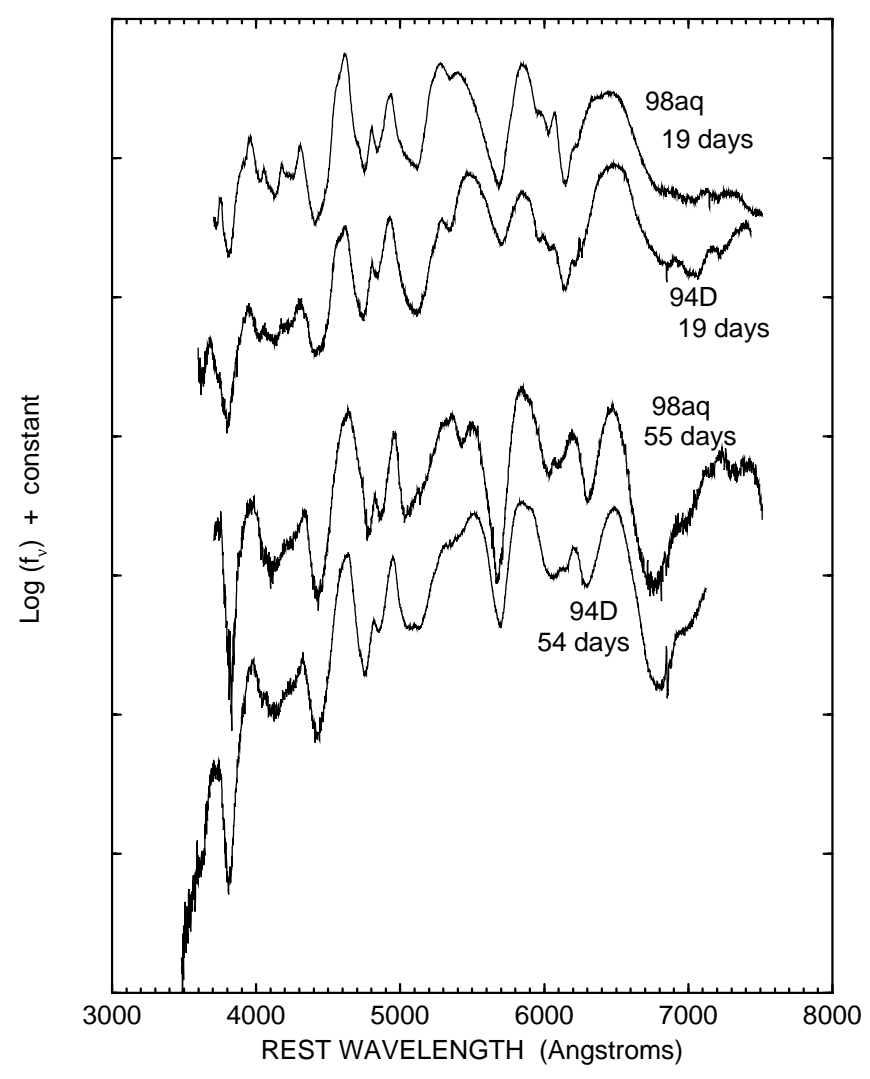

FIG. $2 b$

FIG. 2.-Comparisons of spectra of SN 1998aq and SN 1994D

compared. Except at the earliest times, when the blueshift is changing rapidly with time and that of SN 1994D is mildly higher than that of SN 1998aq, the differences are within the measurement errors of about $250 \mathrm{~km} \mathrm{~s}^{-1}$. Figure 4 shows the blueshifts of four absorption features in SN 1998aq that are not badly blended and for which we are confident of the identifications ( $\mathrm{Si}$ II $\lambda 6355, \mathrm{Ca}$ II $\lambda 3945, \mathrm{~S}$ II $\lambda 5654$, and $\mathrm{Si}$ III $\lambda 4560)$. The blueshifts of the deep Ca II and Si II absorption lines remain near $10,000 \mathrm{~km} \mathrm{~s}^{-1}$, even at 35 days, but those of the weaker Si III and S II features decrease more rapidly.

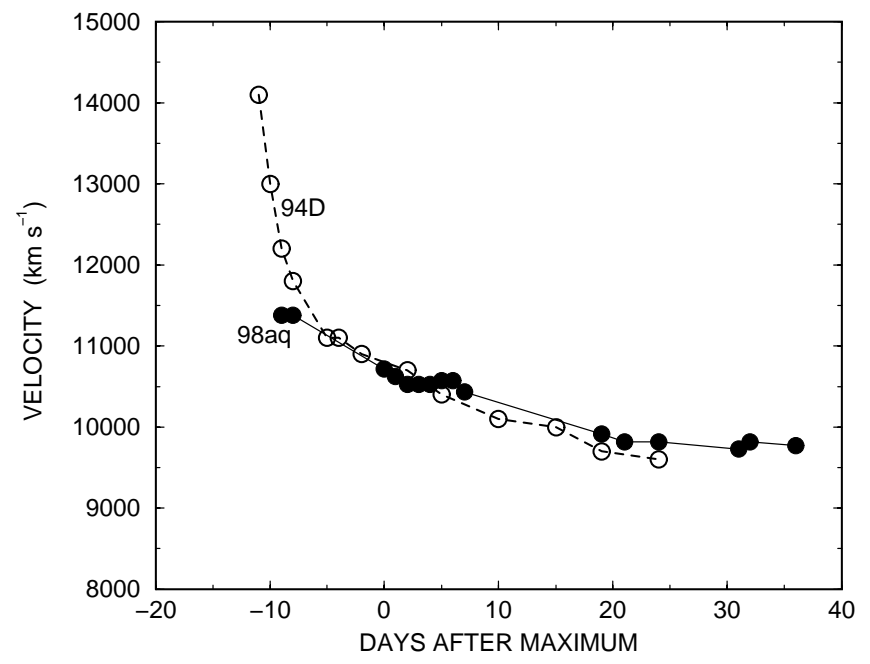

FIG. 3. - Comparison of Si II blueshifts in SN 1998aq and SN 1994D
From Figure 3 we estimate a value of $10,300 \mathrm{~km} \mathrm{~s}^{-1}$ for the $v_{10}(\mathrm{Si}$ II) parameter-the blueshift of the deep Si II absorption 10 days after maximum brightness (Branch \& van den Bergh 1993). In the spectra from days 0, 1, 2, and 3 we measure values of $0.22,0.20,0.22$, and 0.24 for the parameter $R$ (Si II) (Nugent et al. 1995), in good agreement with the value of $0.22 \pm 0.02$ measured by Vinkó et al. (1999) in a day -5 spectrum; we recommend $R(\mathrm{Si}$ II $)=$ $0.22 \pm 0.01$. Hatano et al. (2000) showed and discussed the diversity among $\mathrm{SNe}$ Ia in a plot of $R\left(\mathrm{Si}\right.$ II) versus $v_{10}(\mathrm{Si}$ II) (their Fig. 1). Our measured values put SN 1998aq in the

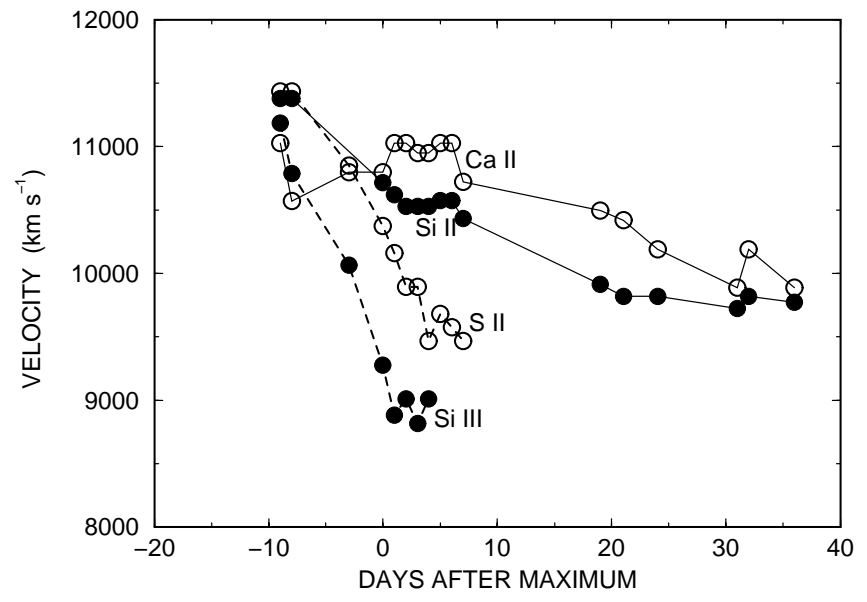

FIG. 4.-Blueshifts of four absorption features in SN 1998aq 
most heavily populated part of their plot, near, e.g., SN 1994D, SN 1996X, SN 1998bu, and SN 1990N. Garnavich et al. (2001) have shown that there is a tight relation between $R$ (Si II) [referred to as $R$ ( $\mathrm{Si}$ II / Ti II) by Garnavich et al.] and $\Delta m_{15}$. Garnavich et al. intended to measure $R(\mathrm{Si}$ II) only in spectra obtained within 3 days of maximum light, but for SN 1998aq their adopted date of maximum differed from the date later reported by Boffi \& Riess (2003). With our recommended value of $R(\mathrm{Si}$ II), based on specta obtained within 3 days of the revised date of maximum, SN 1998aq fits the Garnavich et al. (2001) relation between $R$ (Si II) and $\Delta m_{15}$ even better.

\section{LINE IDENTIFICATIONS IN EARLY SPECTRA}

The analysis of supernova spectra begins with the often difficult process of making line identifications. Only a limited number of good SN Ia spectra have been obtained well before the time of maximum light, so we are especially interested in exploring line identifications in the earliest spectrum of SN 1998aq, at -9 days. For comparison we will also look at the days 0 and 7 spectra. These three spectra are compared in Figure 5. The overall appearance of these spectra is rather similar, although some significant changes do occur during this time interval: the Si II, S II, and Ca II absorptions become deeper with time; the absorption trough from 4800 to $5000 \AA$ in the day -9 spectrum breaks up in the day 7 spectrum; and the absorption trough from 4200 to $4500 \AA$ in the day 7 spectrum is more broken up in the day -9 spectrum.

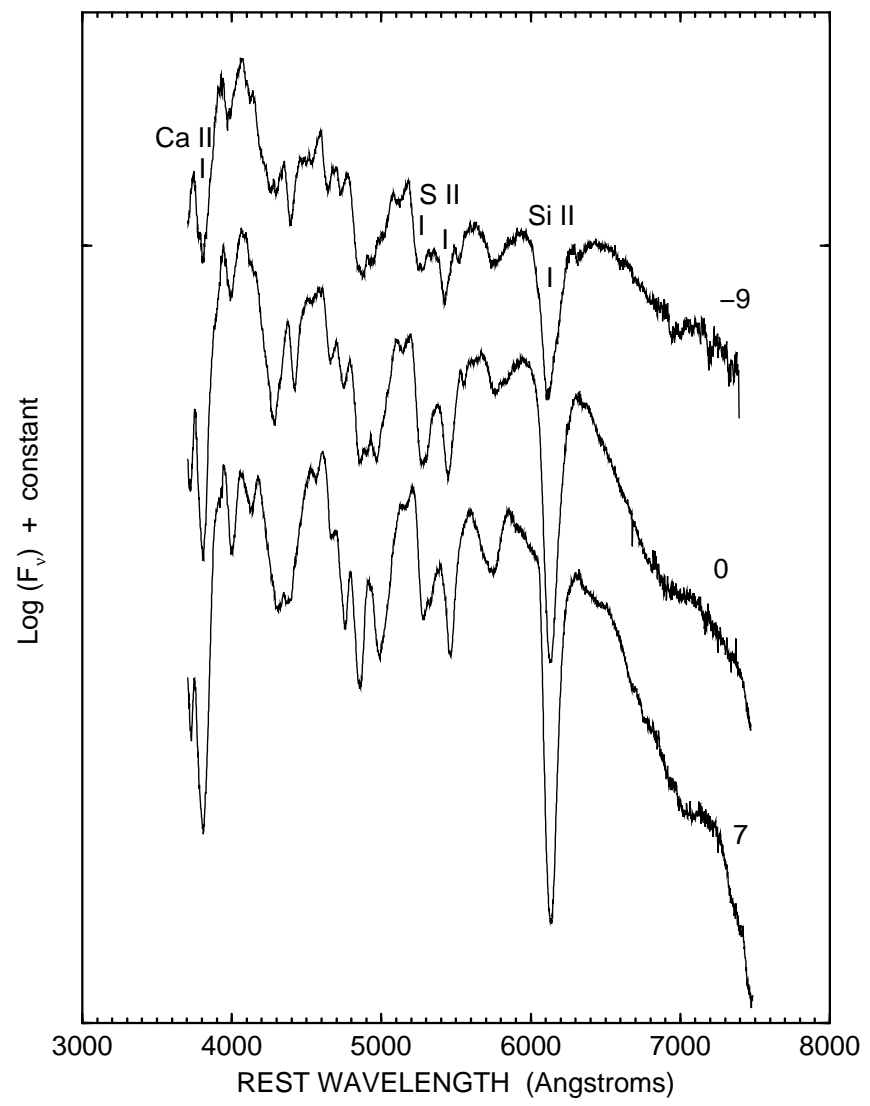

FIG. 5.-Three early spectra of SN $1998 \mathrm{aq}$
We have studied line identifications by comparing the three spectra of Figure 5 with numerous synthetic spectra generated with the parameterized supernova syntheticspectrum code SYNOW. This code has been used and described in several recent papers, e.g., Branch et al. (2002). The basic assumptions are spherical symmetry, a sharp photosphere, velocity proportional to radius, and resonance scattering in the Sobolev approximation. The shape of the underlying continuum is that of a blackbody at temperature $T_{\mathrm{bb}}$. Line optical depths are taken to vary as $\exp \left(-v / v_{e}\right)$, with $v_{e}=1000 \mathrm{~km} \mathrm{~s}^{-1}$ for all synthetic spectra shown in this paper, and no maximuum velocities are imposed. For each ion whose lines are introduced, the maximum Sobolev optical depth of a reference line is a fitting parameter. Ordinarily, the maximum line optical depth is at the photosphere, but for a "detached" ion the line optical depth is zero at the photosphere and rises discontinuously to its maximum value at some higher detachment velocity. The optical depths of the other lines of an ion, relative to that of the reference line, are calculated for Boltzmann excitation at temperature $T_{\mathrm{exc}}$. In this paper, instead of adopting a common value of the excitation temperature $T_{\text {exc }}$ as the default value, the default value for each ion is the temperature at which the ion's reference-line optical depth reaches a maximum in plots of optical depth versus temperature (Hatano et al. 1999b). This is a reasonable default since, e.g., if lines of high excitation are present, they are likely to be formed in high-temperature regions. The default excitation temperatures are used throughout this paper except in one instance that is mentioned below.

\subsection{The Day -9 Spectrum}

The day -9 spectrum is shown in Figure 6, with the measured wavelengths of absorption features labeled, so that we can conveniently refer to each absorption throughout this subsection. In this and all subsequent figures of this paper, instead of plotting $f_{\nu}$ on the vertical axis we plot $f_{\nu} / \nu$, to make the spectrum approximately flat. This provides a better view of features at the longer wavelengths where the underlying continuum is low, which is helpful because the diagnostic value of a spectral feature is not proportional to the level of the underlying continuum. Note also that in

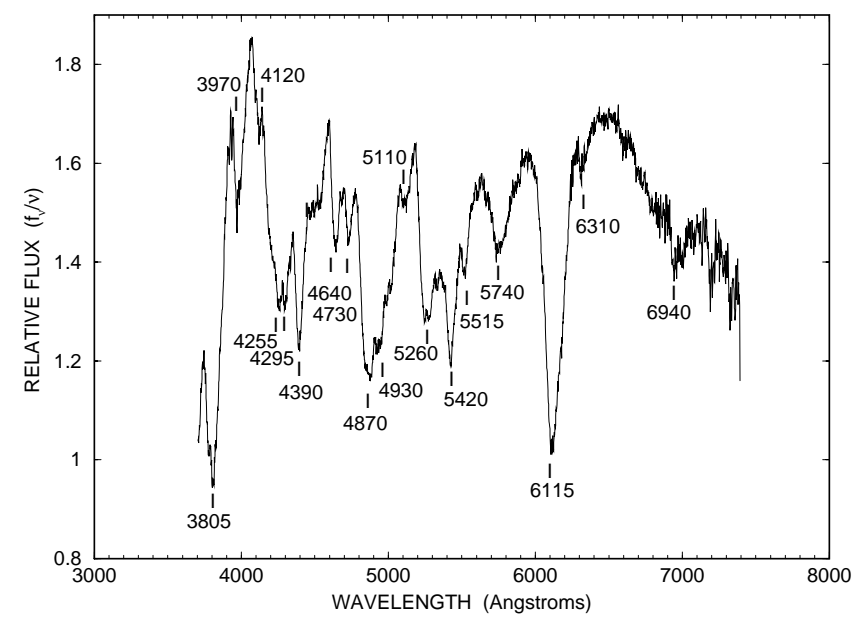

FIG. 6.-Absorption features in the day -9 spectrum of SN 1998aq Note that in this and subsequent figures the vertical axis is $f_{\nu} / \nu$. 


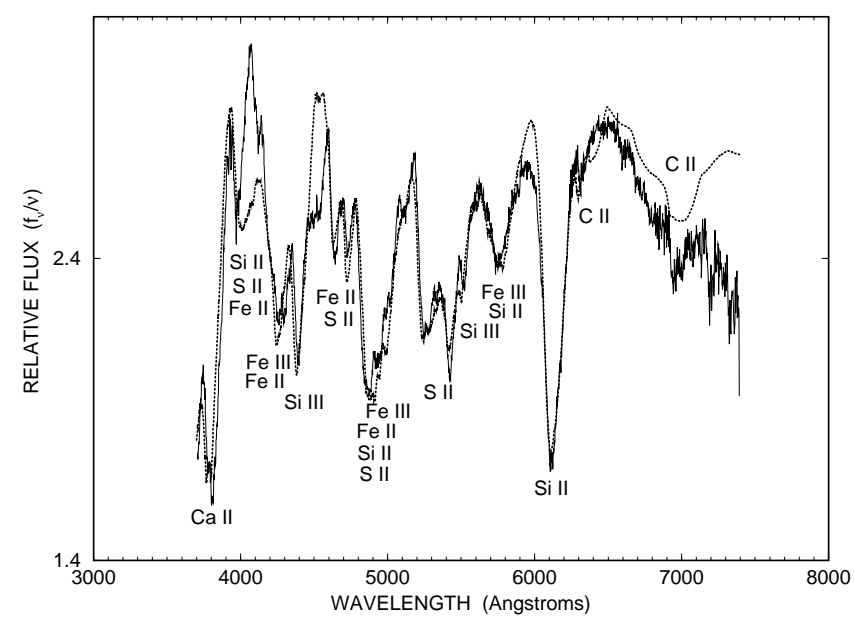

FIG. 7.-Comparison of the day -9 spectrum of SN 1998aq with a SYNOW synthetic spectrum that has $v_{\text {phot }}=13,000 \mathrm{~km} \mathrm{~s}^{-1}, T_{\mathrm{bb}}=18,000$ $\mathrm{K}$, and contains lines of seven ions.

order to show as much detail as possible, in this and subsequent figures, the flux axis does not go to zero.

In Figure 7 the same day -9 spectrum is compared with a synthetic spectrum that has $v_{\text {phot }}=13,000 \mathrm{~km} \mathrm{~s}^{-1}$, $T_{\mathrm{bb}}=16,000 \mathrm{~K}$, and includes lines of seven ions. The ionspecific parameters of the synthetic spectrum are listed in Table 2. The fit shown in Figure 7 is good by supernova standards, although there are two significant discrepancies that we have not yet resolved: the synthetic spectrum is too high from 4460 to $4580 \AA$ and too low from 4000 to $4160 \AA$.

To illustrate the contributions of each ion to the synthetic spectrum, Figures 8-14 are like Figure 7 but with nonzero optical depths of one ion at a time. Owing to multiple scattering, the full synthetic spectrum of Figure 7 is more complex than a simple linear combination of these single-ion spectra.

Figure 8 shows that the main contribution of $\mathrm{Si}$ II is that $\lambda 6355$ accounts for almost all of the $6115 \AA$ absorption. Note that $\lambda 6355$ produces a synthetic emission peak where an absorption feature is observed. The $\lambda 5972$ and $\lambda 5051$ lines contribute to the 5740 and $4870 \AA$ absorptions. The $\lambda 4130$ line contributes to the $3970 \AA$ absorption, but absorption by $\lambda 4130$ is also partially responsible for our failure to fit the observed emission peak near $4070 \AA$. In any case, the presence of $\mathrm{Si}$ II $\lambda 6355$ in the day -9 spectrum of SN 1998aq is definite.

Figure 9 shows that the main contribution of $\mathrm{S}$ II is that $\lambda \lambda 5654$ and 5468 produce the 5420 and $5260 \AA$ absorptions. Lines near $6305 \AA$ make a minor contribution to the $6115 \AA$

TABLE 2

INPUT PARAMETERS FOR Figure 7

\begin{tabular}{|c|c|c|c|}
\hline Line & $\begin{array}{c}\tau \\
\text { (ref) }\end{array}$ & $\begin{array}{c}v_{\min } \\
\left(\mathrm{km} \mathrm{s}^{-1}\right)\end{array}$ & $\begin{array}{l}T_{\text {exc }} \\
(\mathrm{K})\end{array}$ \\
\hline Si II $\lambda 6347$. & 3.5 & 13000 & 8000 \\
\hline $\mathrm{S}_{\text {II }} \lambda 5454 \ldots \ldots \ldots \ldots . . .$. & 1.6 & 13000 & 10000 \\
\hline 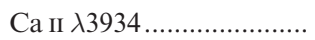 & 6.0 & 13000 & 5000 \\
\hline Si III $\lambda 4553$ & 3.0 & 13000 & 14000 \\
\hline Fe III $\lambda 5156 \ldots \ldots \ldots \ldots \ldots$ & 1.3 & 13000 & 16000 \\
\hline Fe II $\lambda 5018 \ldots \ldots \ldots \ldots \ldots \ldots$ & 0.4 & 20000 & 7000 \\
\hline 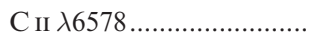 & 0.9 & 14000 & 12000 \\
\hline
\end{tabular}

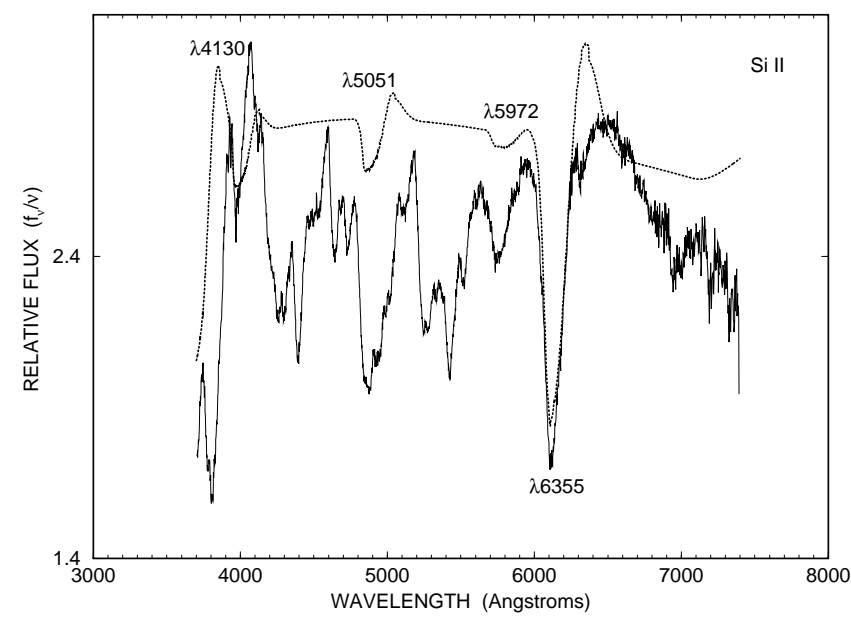

FIG. 8.- Same as Fig. 7, but with only lines of $\mathrm{Si}$ II in the synthetic spectrum.

absorption. The $\lambda 5208$ line may be responsible for the 5110 $\AA$ absorption, although it does not produce a distinct absorption in the full synthetic spectrum of Figure 7. The $\lambda 5032$ line contributes to the $4870 \AA$ absorption and some weaker $\mathrm{S}$ II lines make minor contributions. The $\lambda 4163$ line is partially responsible for our failure to fit the observed emission peak near $4070 \AA$. Nevertheless, the presence of $\mathrm{S}_{\text {II }} \lambda \lambda 5654$ and 5468 is definite.

Figure 10 shows that the only contribution of $\mathrm{Ca}$ II (since the spectrum does not extend to the infrared triplet) is that $\lambda 3945$ (the $\mathrm{H}$ and $\mathrm{K}$ lines) produces the $3805 \AA$ absorption. The presence of Ca II $\lambda 3945$ is definite.

Figure 11 shows that Si III $\lambda 4560$ produces the $4390 \AA$ absorption. It is likely that $\lambda 5743$ is responsible for the 5515 A absorption, although in Figure 7 the synthetic absorption is at a slightly shorter wavelength that the observed one. We are confident of the presence of Si III $\lambda 4560$.

Figure 12 shows that Fe III $\lambda \lambda 5156$ and 4420 are largely responsible for the 4930 and $4255 \AA_{0}$ absorptions. Lines near $6000 \AA$ also contribute to the $5740 \AA$ absorption. This is the one instance in this paper where we depart from the default excitation temperature. At the Fe III default value of 14,000 $\mathrm{K}$ the feature produced by $\lambda 6000$ is present but weaker. We use $16,000 \mathrm{~K}$ to show that Fe III might be mainly responsible

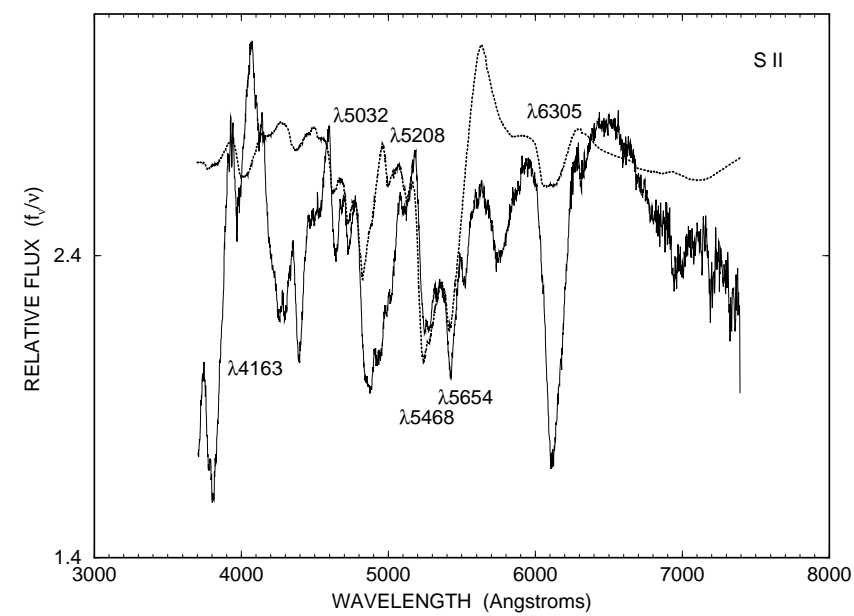

FIG. 9.- Same as Fig. 7, but with only lines of $\mathrm{S}$ II in the synthetic spectrum. 


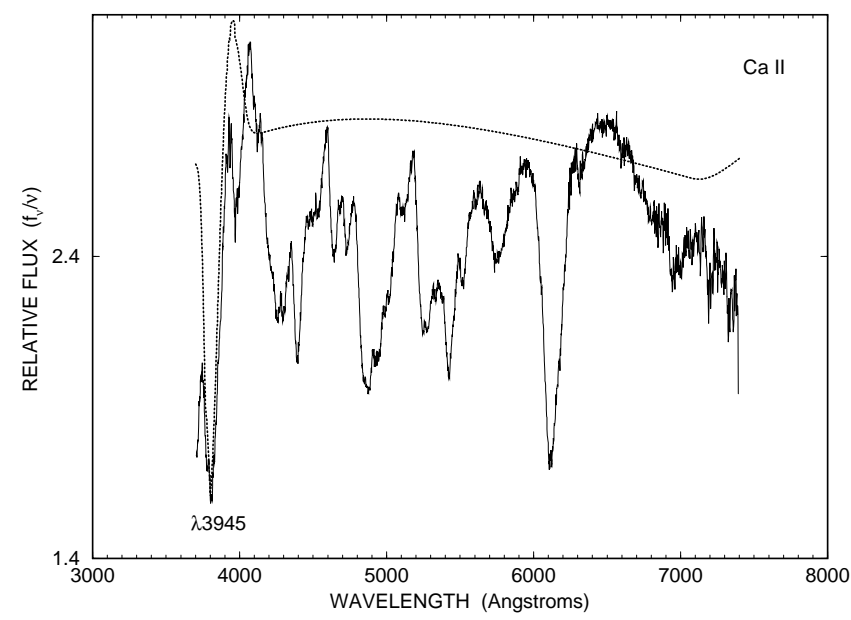

FIG. 10.-Same as Fig. 7, but with only lines of $\mathrm{Ca}$ II in the synthetic spectrum.

for the $5740 \AA$ absorption at this epoch. In any case, we are confident of the presence of Fe III $\lambda \lambda 5156$ and 4420.

Figure 13 shows the contribution of Fe II lines, detached at $20,000 \mathrm{~km} \mathrm{~s}^{-1}$ in order to get the best fit. This means that in the synthetic spectrum, the Fe II optical depths are zero between the velocity at the photosphere, $13,000 \mathrm{~km} \mathrm{~s}^{-1}$, and the detachment velocity of $20,000 \mathrm{~km} \mathrm{~s}^{-1}$. As illustrated in Figure 24 of Branch et al. (2002), the profile of a detached line has a flat-topped emission component and a sharp red edge to the absorption component at the detachment velocity. The presence of detached, high-velocity Fe II in SN 1994D was suggested by Hatano et al. (1999a) on the basis of SYNOW synthetic spectra and then supported by Lentz et al. (2001b) who carried out non-LTE (NLTE) calculations with the PHOENIX code. In Figure 13, we see that Fe II $\lambda \lambda 5169,5018$, and 4924 are contributing to the 4870, 4730 , and $4640 \AA$ absorptions, respectively. Lines near $\lambda \lambda 4549$ and 4025 contribute to the 4295 and $3970 \AA$ absorptions. High-velocity Fe II makes a positive contribution to the full fit in Figure 7, and we consider its presence in the observed spectrum to be likely.

Figure 14 shows the contributions of $\mathrm{C}$ II lines, slightly detached at $14,000 \mathrm{~km} \mathrm{~s}^{-1}$. The strongest line, $\lambda 6580$, plays

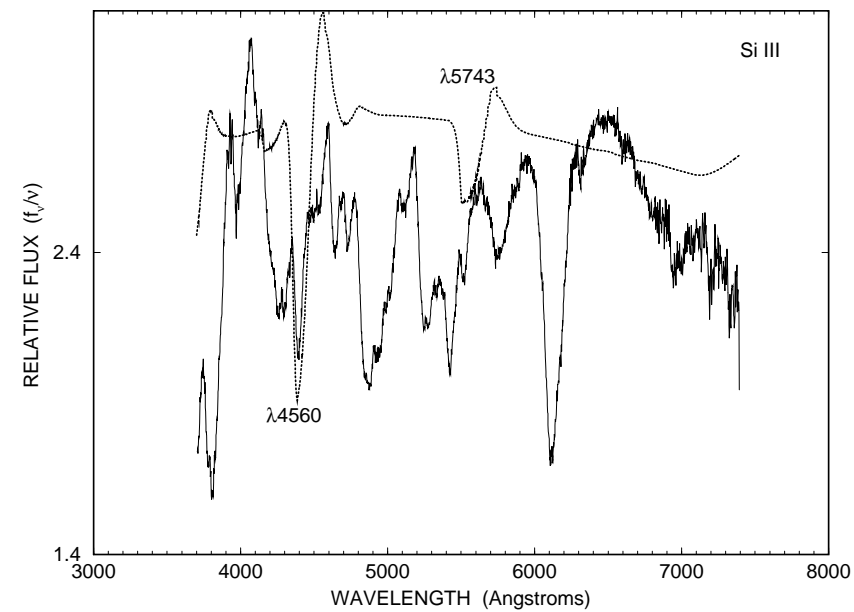

FIG. 11.- Same as Fig. 7, but with only lines of Si III in the synthetic spectrum.

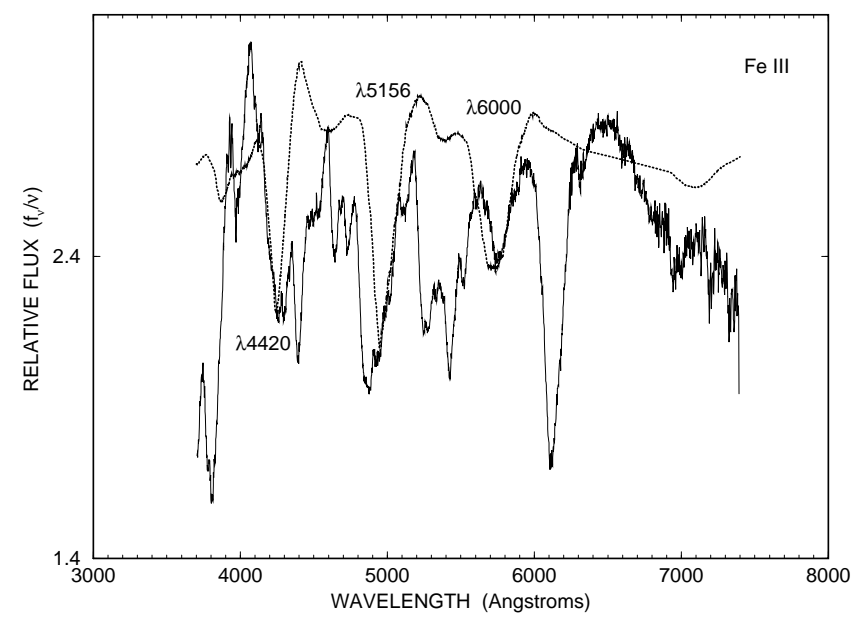

FIg. 12.- Same as Fig. 7, but with only lines of Fe III in the synthetic spectrum.

an important role in the synthetic spectrum: it beats down the unwanted emission component of $\mathrm{Si}$ II $\lambda 6355$ and combines with it in the full synthetic spectrum of Figure 7 to produce a feature near the observed $6310 \AA$ absorption. The $\lambda 7234$ line produces the $6940 \AA$ absorption. (Fig. 14 is a good example of the benefit of plotting $f_{\nu} / \nu$ : it gives a better view of the features produced by $\lambda 6580$ and $\lambda 7234$.) The $\lambda 4267$ line is at the right wavelength to be consistent with the $4120 \AA$ absorption, although $\lambda 4267$ does not produce a distinct feature in the full synthetic spectrum of Figure 7. We consider the presence of $\mathrm{C}$ II lines to be likely.

\subsection{The Days 0 and 7 Spectra}

In Figure 15 the day 0 spectrum of SN 1998aq is compared with a synthetic spectrum that has $v_{\text {phot }}=11,000 \mathrm{~km}$ $\mathrm{s}^{-1}, T_{\mathrm{bb}}=16,000 \mathrm{~K}$, and contains lines of the same seven ions that were used for the day -9 spectrum. The ionspecific parameters of the synthetic spectrum are listed in Table 3. The $\mathrm{Fe}$ II lines now are detached at 18,000 rather than $20,000 \mathrm{~km} \mathrm{~s}^{-1}$, and $\mathrm{Si}$ II and $\mathrm{S}$ II are mildly detached at $12,000 \mathrm{~km} \mathrm{~s}^{-1}$. Compared with the synthetic spectrum for day -9 , the Ca II-line optical depths are increased by a factor of 5 , those of Si II and Fe III are up by small factors, the Fe II optical depths are unchanged, and those of S II, Si III,

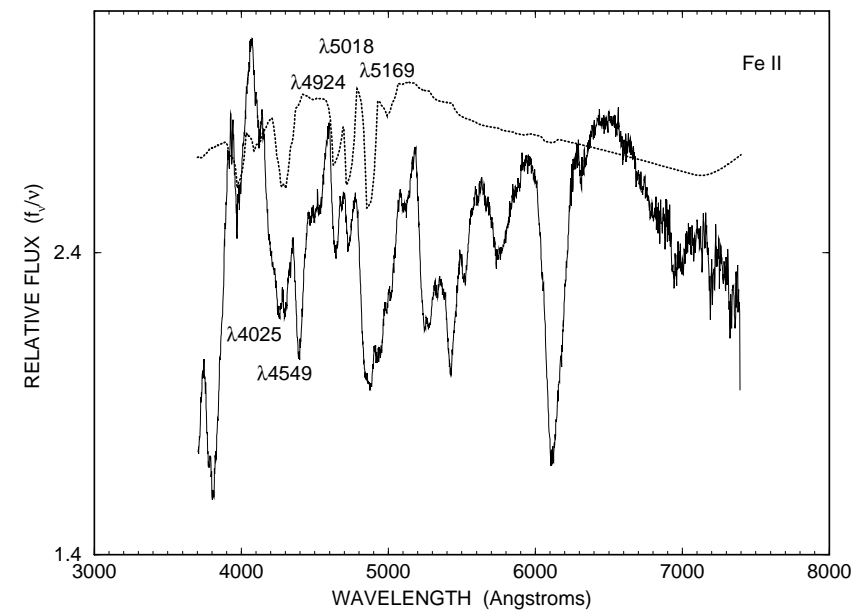

FIG. 13.- Same as Fig. 7, but with only lines of Fe II in the synthetic spectrum. 


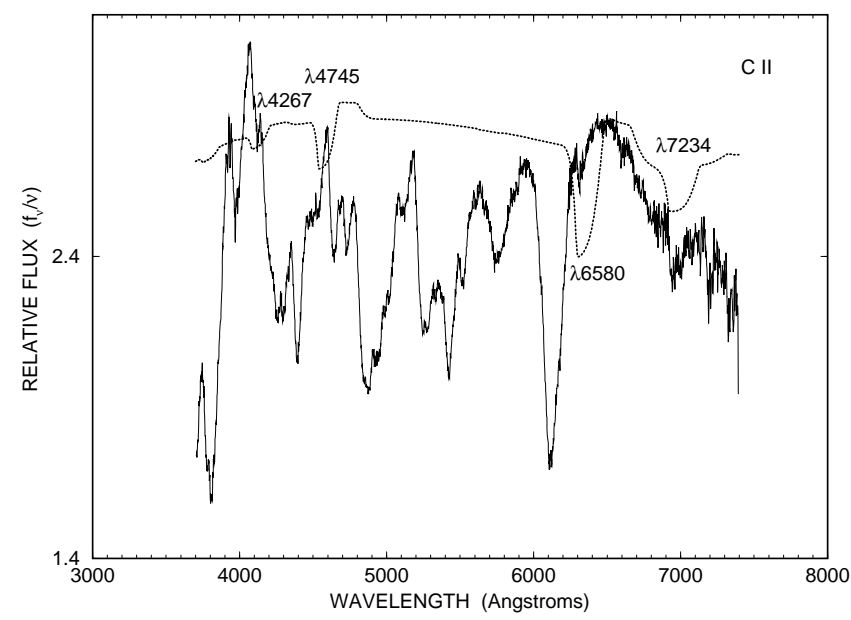

FIG. 14.- Same as Fig. 7, but with only lines of $\mathrm{C}$ II in the synthetic spectrum.

and $\mathrm{C}$ II are down by small factors. The overall fit in Figure 15 is good, the main discrepancies being that the synthetic spectrum again lacks the $4070 \AA$ emission peak, and now the synthetic spectrum is too low from 5800 to $6000 \AA$. The difference in the heights of the apparent continuum levels at wavelengths longer than $6500 \mathrm{~A}$ has been encountered in previous SYNOW studies of $\mathrm{SNe}$ Ia and can be attributed to our use of a blackbody continuum. Obtaining a good fit to the overall energy distribution is a task for detailed spectrum calculations, such as those of Lentz et al. (2001b).

In Figure 15, C II $\lambda 6580$ again beats down the Si II $\lambda 6355$ emission and produces an absorption notch that may correspond to an observed feature. The $\lambda 7234$ line produces a wiggle in the synthetic spectrum that may be at least partially responsible for the knee in the observed spectrum near $7000 \AA$.

In Figure 16 the day 7 spectrum of SN 1998aq is compared with a synthetic spectrum that has $v_{\text {phot }}=11,000 \mathrm{~km}$ $\mathrm{s}^{-1}$ and $T_{\mathrm{bb}}=16,000 \mathrm{~K}$. The ion-specific parameters of the synthetic spectrum are listed in Table 4 . This synthetic spectrum contains lines of six of the seven ions used previously,

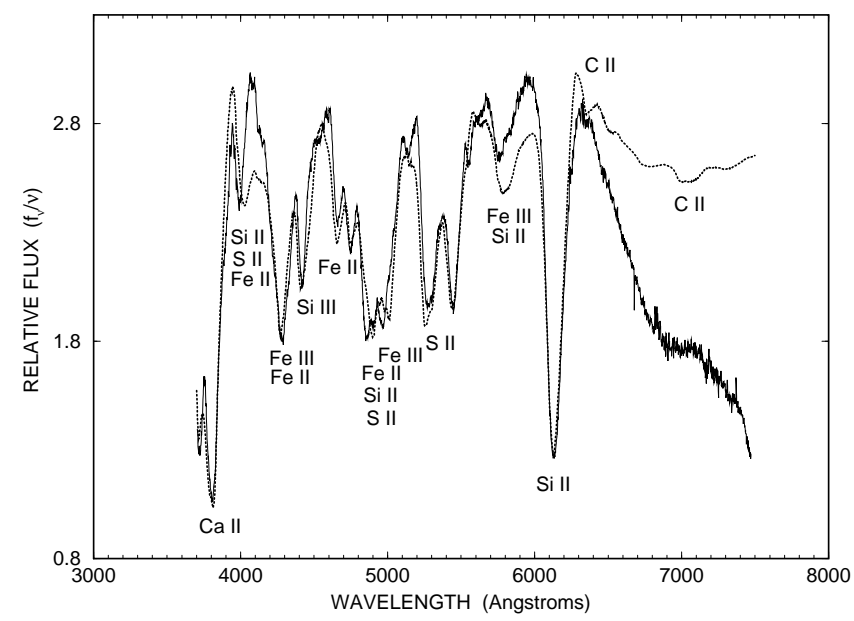

FIG. 15.-Comparison of the day 0 spectrum of SN 1998aq with a SYNOW synthetic spectrum that has $v_{\text {phot }}=11,000 \mathrm{~km} \mathrm{~s}^{-1}, T_{\mathrm{bb}}=16,000$ $\mathrm{K}$, and contains lines of seven ions.
TABLE 3

InPUt PARAMETERS For Figure 15

\begin{tabular}{|c|c|c|c|}
\hline Line & $\begin{array}{c}\tau \\
\text { (ref) }\end{array}$ & $\begin{array}{c}v_{\min } \\
\left(\mathrm{km} \mathrm{s}^{-1}\right)\end{array}$ & $\begin{array}{l}T_{\text {exc }} \\
(\mathrm{K})\end{array}$ \\
\hline 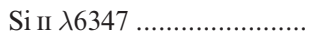 & 4.0 & 12000 & 8000 \\
\hline $\mathrm{S}_{\text {II }} \lambda 5454 \ldots \ldots \ldots \ldots \ldots \ldots$ & 1.5 & 12000 & 10000 \\
\hline Ca II $\lambda 3934 \ldots \ldots \ldots \ldots \ldots$ & 30 & 11000 & 5000 \\
\hline Si III $\lambda 4553$ & 2.2 & 11000 & 14000 \\
\hline 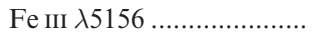 & 1.7 & 11000 & 14000 \\
\hline Fe II $\lambda 5018 \ldots \ldots \ldots \ldots \ldots \ldots$ & 0.4 & 18000 & 7000 \\
\hline 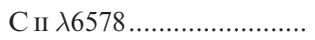 & 0.5 & 11000 & 12000 \\
\hline
\end{tabular}

Si III now being omitted. Fe II has two components: one detached at $18,000 \mathrm{~km} \mathrm{~s}^{-1}$, as in the day 0 spectrum, and now also an undetached component. Compared to the day 0 spectrum, the optical depths of the Si II, S II, Ca II, C II, and $\mathrm{Fe}$ II lines have been increased, while Fe III has been decreased. The synthetic spectrum of Figure 16 also includes contributions from Mg II, Na I, Co II, and Ti II lines, but their contributions are not major, and we do not consider their presence in the observed spectrum to be established. To the extent to which we have been able to account for it, the breakup of the 4800-5000 $\AA$ region and the filling in of the 4200-4500 $\AA$ region, both mentioned above in the discussion of Figure 5, are caused by the emergence of the undetached Fe II component. Apart from the problem of the high synthetic continuum at wavelengths greater than $6600 \mathrm{~A}$, the main discrepancy in Figure 16 is that the synthetic spectrum is too low in the interval 5100-6000 .

In Figure 16, C II $\lambda 6580$ leads to a (perhaps fortuitously) good fit at the top of the Si II $\lambda 6355$ emission, and again $\lambda 7234$ produces a feature that appears to be responsible for the one observed near $7000 \AA$.

\section{DISCUSSION}

The identification of lines of Si II, S II, Ca II, Fe III, and $\mathrm{Si}$ III in early spectra of SNe Ia is not new, but the figures in this paper clearly illustrate the extent to which lines of these ions can account, with simple assumptions, for the observed spectral features in SN 1998aq. It is instructive to compare

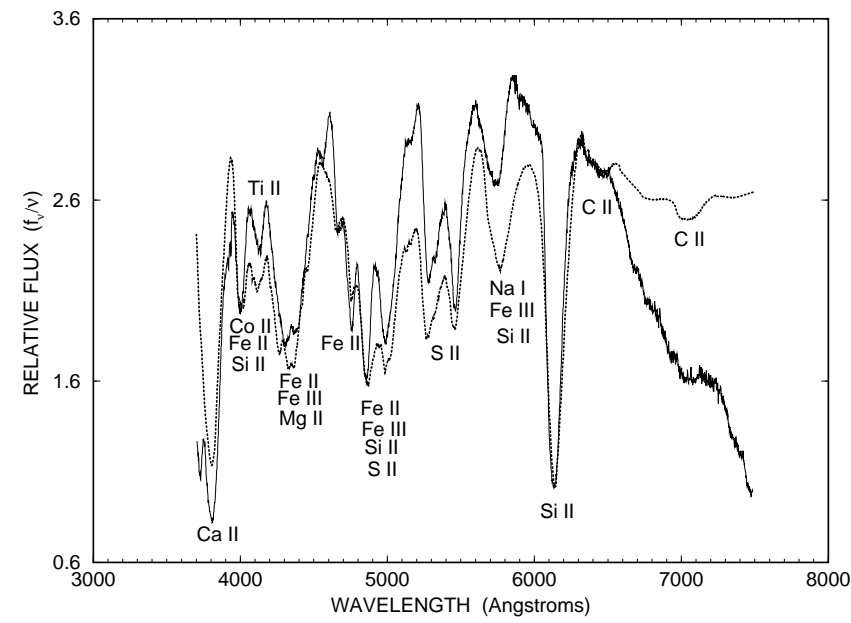

FIG. 16.-Comparison of the day 7 spectrum of SN 1998aq with a SYNOW synthetic spectrum that has $v_{\text {phot }}=11,000 \mathrm{~km} \mathrm{~s}^{-1}, T_{\mathrm{bb}}=16,000$ $\mathrm{K}$, and contains lines of 10 ions. 
TABLE 4

INPUT PARAMETERS FOR Figure 16

\begin{tabular}{|c|c|c|c|}
\hline Line & $\begin{array}{c}\tau \\
\text { (ref) }\end{array}$ & $\begin{array}{c}v_{\min } \\
\left(\mathrm{km} \mathrm{s}^{-1}\right)\end{array}$ & $\begin{array}{l}T_{\text {exc }} \\
(\mathrm{K})\end{array}$ \\
\hline Si II $\lambda 6347 \ldots$. & 12 & 11000 & 8000 \\
\hline 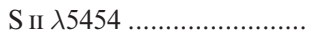 & 1.8 & 11000 & 10000 \\
\hline 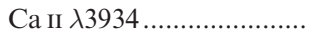 & 40 & 11000 & 5000 \\
\hline Fe III $\lambda 5156 \ldots \ldots \ldots \ldots \ldots \ldots . . . . . . . . .$. & 1.2 & 11000 & 14000 \\
\hline Fe II $\lambda 5018 \ldots \ldots \ldots \ldots \ldots \ldots . . . . . . . . .$. & 2.0 & 11000 & 7000 \\
\hline 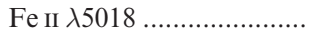 & 2.0 & 18000 & 7000 \\
\hline 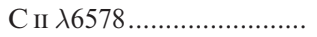 & 0.9 & 11000 & 12000 \\
\hline 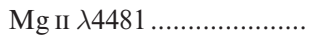 & 1.0 & 11000 & 12000 \\
\hline 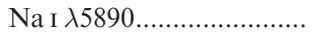 & 0.5 & 11000 & 12000 \\
\hline 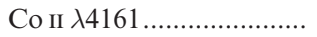 & 1.0 & 14000 & 12000 \\
\hline 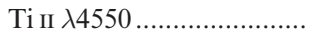 & 0.2 & 14000 & 6000 \\
\hline
\end{tabular}

parameterized SYNOW spectrum fits with NLTE synthetic spectra for specific explosion models. Lentz et al. (2001b) used the PHOENIX code to make detailed calculations of spectra of the deflagration model W7 (Nomoto, Thielemann, \& Yokoi 1984) and found reasonably good agreement with the early spectra of SN 1994D, which as we have seen in $\S 2$ was spectroscopically similar to SN 1998aq. Figure 17 is like Figure 7 but also including the PHOENIX spectrum of model W7 at 11 days after explosion (corresponding to about 9 days before maximum light). The model W7 spectrum does have peaks and dips at some of the right wavelengths, but there is room for improvement. For example, the W7 spectrum lacks the 4390 A absorption that we attribute to $\mathrm{Si}$ III $\lambda 4560$, and the $\mathrm{W} 7$ emission peaks near 5900 and $6400 \AA$ are too high. Ongoing work involving close comparative studies of observed spectra, SYNOWlevel synthetic spectra, and PHOENIX-level spectra of explosion models will provide indications of how explosion models need to be altered to give better agreement with observed spectra.

Apparently, $\mathrm{Fe}$ II features forming at high velocity, $\geq 18,000 \mathrm{~km} \mathrm{~s}^{-1}$, are present at the three epochs we have studied. Hatano et al. (1999a) identified high-velocity $\left(\geq 20,000 \mathrm{~km} \mathrm{~s}^{-1}\right) \mathrm{Ca}$ II and $\mathrm{Fe}$ II lines in SN 1994D. Kasen

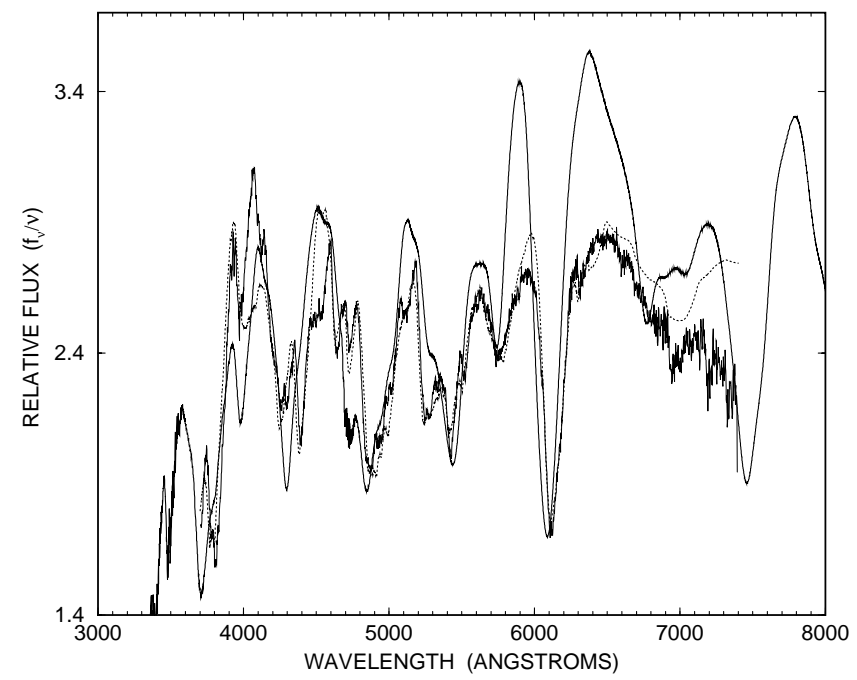

FIG. 17.- Same as Fig. 7, but including a PHOENIX synthetic spectrum (smooth solid line) for model W7 at 11 days after explosion, from Lentz et al. (2001b). et al. (2003) discuss high-velocity $\left(18,000-25,000 \mathrm{~km} \mathrm{~s}^{-1}\right)$ components of the Ca II infrared triplet in flux and polarization spectra of SNe 2001el, and Thomas et al. (2003) discuss high-velocity $\left(\geq 23,000 \mathrm{~km} \mathrm{~s}^{-1}\right) \mathrm{Ca}$ II in flux spectra of the peculiar SN 2000cx. Both Kasen et al. (2003) and Thomas et al. (2003) conclude that the Ca II features formed in nonspherical geometry, probably in clumps located in front of the photosphere. The spectra of SN 1998aq do not extend to the $\mathrm{Ca}$ II infrared triplet (and barely extend to the highvelocity side of the $\mathrm{Ca}$ II $\mathrm{H}$ and $\mathrm{K}$ feature) so we have not established the presence of high-velocity $\mathrm{Ca}$ II features in SN 1998aq. In any case, the presence of high-velocity features in early spectra of SNe Ia is evidently not uncommon. Comparative studies of early-time polarization and flux spectra of SNe Ia, to clarify the composition, geometry, and origin of the high-velocity matter, are underway. It will be interesting to see how much of the spectroscopic and (owing to differences in line blocking) photometric diversity among $\mathrm{SNe}$ Ia, including even some of the postmaximum diversity, is caused by spectral features forming at high velocity.

Perhaps the most significant result of our analysis of SN 1998aq is the evidence of C II lines. The possible presence of C II in early spectra of SNe Ia has been discussed before. For instance, Mazzali (2001) tentatively attributed absorptions in a day -14 spectrum of SN $1990 \mathrm{~N}$ to C II $\lambda \lambda 6580$ and 7234 forming near the photospheric velocity of about $16,000 \mathrm{~km} \mathrm{~s}^{-1}$. The features that we attribute to $\lambda \lambda 6580$ and 7234 in SN 1998aq are conspicuous in our figures because we plot $f_{\nu} / \nu$ to raise the red end of the spectra. If C II is present in SN 1998aq, then it is also present in at least some of the other SNe Ia that have been observed at early times; e.g., similar features can be seen in spectra of SN 1994D (Fig. 2) and SN 1996X (Salvo et al. 2001).

The presence of C II would have important implications for SN Ia explosion models. In the synthetic spectrum of Figure 7, we use C II detached at $14,000 \mathrm{~km} \mathrm{~s}^{-1}$, but in Figures 15 and 16, $\mathrm{C}$ II is undetached and therefore forming down to the photospheric velocity of $11,000 \mathrm{~km} \mathrm{~s}^{-1}$ (and we have not established the absence of carbon at even lower velocities). Published delayed-detonation models for normal SNe Ia have very little carbon below about 30,000 km $\mathrm{s}^{-1}$. Model W7, a parameterized one-dimensional deflagration model, has unburned carbon down to $14,000 \mathrm{~km} \mathrm{~s}^{-1}$. Recently computed three-dimensional deflagrations (Khokhlov 2000; Gamezo et al. 2003) have carbon at all ejection velocities. The spectroscopic appearance of such models has begun to be explored (Baron et al. 2003). In the context of existing models, the presence of $\mathrm{C}$ II lines may be consistent with deflagrations but not with delayed detonations. However, the spectra of three-dimensional deflagration models appear to be inconsistent with SN Ia spectra in other respects (Thomas et al. 2002; Gamezo et al. 2003), while delayed-detonation models have been found to be consistent with certain unusual $\mathrm{SNe}$ Ia (Lentz et al. 2001a) and in some respects with normal SNe Ia (Wheeler et al 1998). Much more work on comparing synthetic spectra, both parameterized and detailed, with observed spectra of $\mathrm{SNe}$ Ia is needed to guide us toward satisfactory explosion models.

We thank the referee, N. Patat, for constructive comments. This work has been supported by NSF grants AST 99-86965 and AST 02-04771 and NASA grant NAG 512127. 


\section{REFERENCES}

Baron, E., Lentz, E. J. \& Hauschildt, P. H. 2003, ApJ, 588, L29

Boffi, F. R., \& Riess, A. G. 2003, in Symbiotic Stars Probing Stellar Evolution, ed. R. L. M. Corradi, J. Mikolajewska, \& T. J. Mahoney (San Francisco: ASP), in press

Branch, D., et al. 2002, ApJ, 566, 1005

Branch, D., \& van den Bergh, S. 1993, AJ, 105, 2231

Fabricant, D., Cheimets, P., Caldwell, N., \& Geary, J. 1998, PASP, 110, 79

Filippenko, A. V. 1982, PASP, 94, 715

Gamezo, V., Khokhlov, A., Oran, E., Chtchelkanova, A., \& Rosenberg, R. 2003, Science, 299, 77

Garnavich, P. M., et al. 2001, ApJ, submitted (astro-ph/0105490)

Garnavich, P., et al. 1998, IAU Circ. No. 6880

Hatano, K., Branch, D., Fisher, A., Baron, E., \& Filippenko, A. V. 1999a, ApJ, 525, 881

Hatano, K., Branch, D., Fisher, A., Millard, J., \& Baron, E. 1999b, ApJS, 121,233

Hatano, K., Branch, D., Lentz, E. J., Baron, E., Filippenko, A. V., \& Garnavich, P. M. 2000, ApJ, 543, L49

Horne, K. 1986, PASP, 98, 609

Hurst, G. M., Armstrong, M., \& Arbour, R. 1998, IAU Circ. No. 6875

Kasen, D., et al. 2003, ApJ, in press

Khokhlov, A. 2000, ApJ, submitted (astro-ph/0008463)

Lentz, E. J., Baron, E., Branch, D., \& Hauschildt, P. H. 2001a, ApJ, 547, 402
Li, W., et al. 2001, ApJ, 546, 734

Matheson, T., Filippenko, A. V., Ho, L. C., Barth, A. J., \& Leonard, D. C. 2000, AJ, 120,1499

Mazzali, P. A. 2001, MNRAS, 321, 341

Nomoto, K., Thielemann, F.-K., \& Yokoi, K. 1984, ApJ, 286, 644

Nugent, P., Phillips, M. M., Baron, E., Branch, D., \& Hauschildt, P. 1995, ApJ, 455, L147

Patat, F., Benetti, S., Cappellaro, E., Danziger, I. J., Della Valle, M., Mazzali, P. M., \& Turatto, M. 1996, MNRAS, 278, 111

Phillips, M. M. 1993, ApJ, 413, L105

Riess, A. G., et al. 2003, in preparation

Saha, A., Sandage, A., Tammann, G. A., Dolphin, A. E., Christensen, J., Panagia, N., \& Macchetto, F. D. 2001, ApJ, 562, 314

Salvo, M. E., Cappellaro, E., Mazzali, P. A., Benetti, S., Danziger, I. J., Patat, F. \& Turatto, M. 2001, MNRAS, 321, 254

Stetson, P. M., \& Gibson, B. K. 2001, MNRAS, 328, 1L

Thomas, R. C., Branch, D., Baron, E., Nomoto, K., Li, W., \& Filippenko, A. V. 2003, ApJ, submitted (astro-ph/0302260)

Thomas, R. C., Kasen, D., Branch, D., \& Baron, E. 2002, ApJ, 567, 1037

Vinkó, J., Kiss, L. L., Thomson, J., Furész, G., Lu, W., Kaszás, G., \& Balog, Z. 1999, A\&A, 345, 592

Wade, R. A., \& Horne, K. D. 1988, ApJ, 324, 411

Wheeler, J. C., Höflich, P., Harkness, R. P., \& Spyromilio, J. 1998, ApJ, 496, 908 